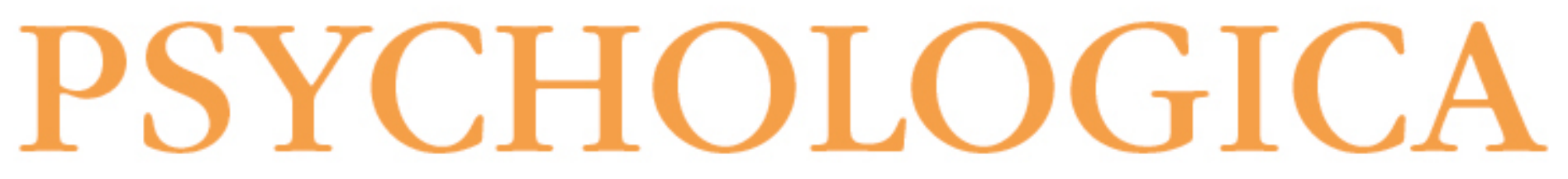

Aristóteles versus Philoponus: um estudo funcional da física intuitiva dos projécteis

Autor(es): $\quad$ Teixeira, Nuno de Sá; Oliveira, Armando Mónica

Publicado por: Imprensa da Universidade de Coimbra

URL

persistente: URI:http://hdl.handle.net/10316.2/3484

DOI: $\quad$ DOl:http://dx.doi.org/10.14195/1647-8606_52-2_23

Accessed : $\quad$ 26-Apr-2023 12:29:27

A navegação consulta e descarregamento dos títulos inseridos nas Bibliotecas Digitais UC Digitalis, UC Pombalina e UC Impactum, pressupõem a aceitação plena e sem reservas dos Termos e Condições de Uso destas Bibliotecas Digitais, disponíveis em https://digitalis.uc.pt/pt-pt/termos.

Conforme exposto nos referidos Termos e Condições de Uso, o descarregamento de títulos de acesso restrito requer uma licença válida de autorização devendo o utilizador aceder ao(s) documento(s) a partir de um endereço de IP da instituição detentora da supramencionada licença.

Ao utilizador é apenas permitido o descarregamento para uso pessoal, pelo que o emprego do(s) título(s) descarregado(s) para outro fim, designadamente comercial, carece de autorização do respetivo autor ou editor da obra.

Na medida em que todas as obras da UC Digitalis se encontram protegidas pelo Código do Direito de Autor e Direitos Conexos e demais legislação aplicável, toda a cópia, parcial ou total, deste documento, nos casos em que é legalmente admitida, deverá conter ou fazer-se acompanhar por este aviso. 


\section{NÚMERO 52}

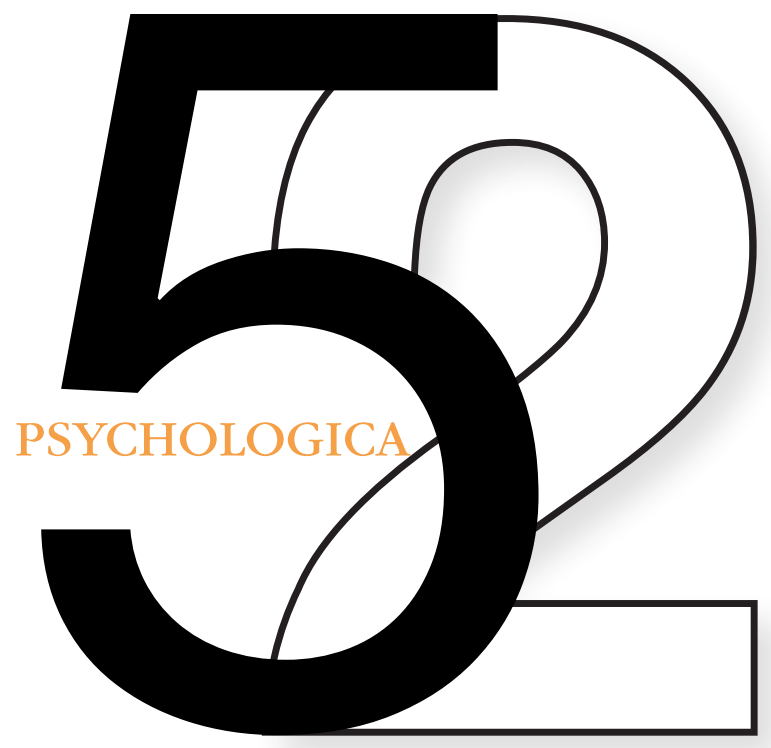

VOLUME II

IMPRENSA DA UNIVERSIDADE DE COIMBRA

FACULDADE DE PSICOLOGIA E DE CIÊNCIAS DA EDUCAÇÃo DA UNIVERSIDADE DE COIMBRA 


\title{
Aristóteles versus Philoponus: um estudo funcional da física intuitiva dos projécteis
}

\author{
Nuno de Sá Teixeira' \& Armando Mónica Oliveira²
}

Enquanto domínio de investigação, a física intuitiva ocupa-se das representações espontâneas do mundo físico e do seu modo funcionamento (em particular, dos eventos mecânicos). Uma linha de estudo influente sugere um paralelismo entre o perfil dos erros diagnosticados nessas representações e as teorias pré-newtonianas, como a física aristotélica ou a física medieval do ímpeto. Neste estudo comparamse duas propostas de matematização da relação entre a velocidade de um móvel, a força aplicada ao projectá-lo e a resistência do meio, a primeira derivada a partir dos escritos de Aristóteles e a segunda proposta por Philoponus no século VI. A metodologia da teoria da Integração de Informação e da Medida Funcional foi utilizada para esse efeito, resultando no estabelecimento de um modelo algébrico divisivo, conforme à proposta aristotélica. O significado deste resultado é discutido, propondo-se uma relação com o quadro de estudo dos modelos mentais. Discutese igualmente o contributo potencial da álgebra cognitiva para a física intuitiva.

PALAVRAS-CHAVE: Física Intuitiva, Física Ingénua, Ímpeto, Modelos Mentais, Teoria da Integração da Informação, Álgebra Cognitiva.

Um adquirido da ciência moderna, manifesto no seu próprio esforço de constituição, é a divergência entre intuição comum e realidade física. Esta divergência suscita duas interrogações: por que são as nossas intuições persistentemente erróneas, ao ponto de se converterem num obstáculo à aprendizagem formal, em domínios nos quais dispomos de uma experiência directa e quotidiana, como o movimento dos corpos e as colisões entre eles (McDermott, 1991)? E por que somos, apesar da natureza fisicamente injustificada de tais intuições, genericamente bem sucedidos na interacção perceptivo-motora com os eventos físicos, uma condição óbvia da nossa sobrevivência enquanto indivíduos e enquanto espécie (Bertamini, Spponer

\footnotetext{
1 Instituto de Psicologia Cognitiva - Universidade de Coimbra - nuno_desateixeira@fpce.uc.pt Trabalho realizado no âmbito do projecto PPCDT/PSI/60769/2004 e da bolsa SFRH/BD/36067/2007, financiados pela Fundação para a Ciência e Tecnologia. 2 Instituto de Psicologia Cognitiva - Universidade de Coimbra
} 
\& Hecht, 2004)? Estas questões animam um domínio de estudo da psicologia cognitiva conhecido pelo nome de "física intuitiva" (McCloskey, 1983a) ou "física ingénua" (Profitt, 1999).

\section{A física intuitiva da mecânica}

Enquanto domínio de investigação, a "física intuitiva" ocupa-se das representações espontâneas sobre a estrutura e modo de funcionamento do mundo físico. Apesar da generalidade desta definição, que não exclui nenhuma classe de fenómenos naturais (e.g., do electromagnetismo ao comportamento dos fluidos e gases, do calor à óptica física), uma atenção especial tem sido dedicada à mecânica clássica (newtoniana), em particular às intuições comuns sobre o movimento de corpos projectados, largados de um referencial inercial, ou animados de movimento circular (Viennot, 1979, Trowbridge \& McDermott, 1980; Champagne, Klopfer \& Anderson, 1980; Caramazza, McCloskey, \& Green, 1981; Clement, 1982; McCloskey, 1983a; 1983b; diSessa, 1988). As Leis de Newton fornecem neste quadro um critério natural de correcção e o ponto de partida para a caracterização das discrepâncias entre julgamentos intuitivos e física formal.

Entre as concepções erróneas mais comuns diagnosticadas por estes trabalhos incluem-se a crença na "queda-a-direito" (straight-down belief) dos corpos largados por um móvel (McCloskey, Washburn, \& Felch, 1983), a crença num ímpeto curvilinear (Mcloskey \& Khol, 1983; Kaiser, Proffitt, \& Anderson, 1985) e a crença de que objectos pesados caem mais depressa do que objectos leves (Champagne et. al., 1980). Perante a prevalência destas concepções, às quais nem os estudantes com instrução formal em física (Clement, 1982) nem os peritos (Hetch \& Profitt, 1995) estão imunes, duas atitudes explicativas principais têm sido adoptadas. Uma delas atribui a inadequação das nossas intuições a limitações de processamento, em particular no que toca à capacidade de integrar diferentes fontes de informação relevantes (Kaiser, Proffit \& Anderson, 1985). Profitt e Gilden (1989) sustentam neste sentido que a apreensão da dinâmica subjacente a um evento físico está fundamentalmente limitada pela nossa incapacidade de derivar quantidades multidimensionais. A segunda assenta no estabelecimento de paralelos epistemológicos entre as nossas intuições e aquelas sistematizadas pelos paradigmas anteriores a Newton, como a física aristotélica (Champagne, Klopfer, L. \& Anderson, 1980) ou a física medieval do ímpeto (McCloskey, 1983a). Os erros documentados são atribuídos neste quadro à influência de teorias ingénuas (globais e coerentes) sobre o movimento que recapitulam, ao nível da cognição individual, diferentes estádios históricos de constituição da mecânica. 


\section{Concepções históricas do movimento dos projécteis}

O traço comum às concepções pré-newtonianas do movimento é a convicção, contrária à primeira lei de Newton, de que todo o movimento, diversamente do estado de repouso, necessita de uma causa (ver Laird \& Roux, 2008). Aristóteles concebeu essa causa como uma força externa, instaurando desse modo uma dualidade entre o móvel (motus) e o agente do seu movimento (movens). Esta concepção suscitava dificuldades particulares ao entendimento do movimento dos projécteis após a separação do lançador (o seu movens), levando Aristóteles a conjecturar um duplo papel do ar, "resistivo" quando à frente do móvel e "motor" ou cinético quando localizado atrás dele (Prescott \& Mitchelmore, 2005).

Uma concepção distinta foi proposta pelo grego Philoponus no século VI, e sistematizada mais tarde pelo parisiense Jean Buridan, no século XIV, que atribuía a causa do movimento a uma força interna ao objecto, o impetus. No caso de um projéctil, esta força era "impressa” ao corpo no acto do lançamento, permitindoIhe deslocar-se na direcção que o lançador fixara ao movimento. Apesar das semelhanças aparentes, o impetus distingue-se fundamentalmente do momentum da mecânica clássica ( $p=m v$ ), que constitui, não uma causa do movimento, e nesse sentido uma propriedade absoluta do móvel, mas uma quantidade relativa utilizada para descrever o movimento num referencial (McCloskey, 1983a).

Para dar conta da circunstância de que um projéctil acaba por se imobilizar, a física medieval incluiu a ideia de uma dissipação progressiva do ímpeto. Essa dissipação foi concebida como espontânea em autores como Avicena (século XI) e como devida à acção de forças externas como a resistência do ar e factores de fricção por Buridan (Jammer, 1957; Rezende \& Barros, 2001). Até à dedução da trajectória parabólica por Galileu (inicialmente ele próprio um aderente da concepção do impetus, a dissipação do ímpeto inspirou diferentes modelos do movimento dos projécteis, dos percursos em $L$ invertido de Avicena às combinações mais complexas de segmentos lineares e secções curvas de Alberto de Saxónia, no século XIV (McCloskey, 1983a).

\section{Primeiros esforços de matematização da relação entre força apli- cada e velocidade do projéctil}

A matematização do movimento dos corpos não é possivel, em sentido próprio, no quadro das físicas qualitativas anteriores a Newton, como a aristotélica (marcada pela diferença entre movimentos naturais e violentos ou o mundo sublunar e supra-lunar) ou a medieval (repousando ainda na diferença de natureza entre estado de repouso e estado de movimento) (cf. Celeyrette, 2008). No entanto, a 
utilização de uma linguagem quantitativa, ainda que para falar de qualidades, constitui um traço de alguns dos desenvolvimentos característicos da física medieval, em particular no século XIV (Clagett, 1957; Thakkar, 2007).

As primeiras formulações algébricas da relação entre força e velocidade do móvel $\mathbf{5 4 8}$ foram derivadas por autores medievais dos enunciados qualitativos de Aristóteles na Física e no De caelo (Celeyrette, 2008). Para Aristóteles todo o movimento ocorria num meio determinado (era impossível no vazio, onde deixaria se ser sucessivo, para se tornar infinito) e dependia, nas suas propriedades, da relação entre a força motriz e a resistência oposta pelo meio ao movimento. Quanto à forma exacta desta relação, Aristóteles parecia sugerir que a velocidade $(V)$ era proporcional à força a plicada $(F)$ e inversamente proporcional à resistência $(R)$, de tal forma que ao duplicar a força se obteria uma duplicação da velocidade, e ao duplicar a resistência se reduziria a metade a velocidade. Em notação algébrica (evidentemente desconhecida nos dias de Aristóteles):

$V=F / R$

Uma formulação distinta foi proposta por Philoponus, proponente da teoria do impetus, defendendo uma proporcionalidade da velocidade ao excesso, avaliado por meio de uma diferença, da força motriz sobre a resistência:

$V=F-R$

Esta expressão, retomada por Avempace no século XI e aceite por Tomás de Aquino, Roger Bacon, ou Duns Scott, evita um resultado paradoxal do enunciado peripatético: a existência de uma velocidade positiva $(=1)$ quando $\mathrm{F}$ e R se equivalem. Foi, por outro lado, criticada por Averróis (século XII), Alberto o Grande ou Egídio de Roma, que defendem o cânone aristotélico (Jammer, 1957). Só através da regra F/R se pode evitar o problema do vazio, argumenta Averróis, já que, no vazio $(R=0)$, a razão F/R não existiria (Celeyrette, 2008).

Uma terceira lei, basicamente em acordo com a posição de Averróis, foi proposta por Bradwardine, no século XIV. O propósito desta "lei" era o de obter uma forma de proporcionalidade da velocidade à razão entre a força e a resistência (F/R) tal que, quando esta razão tendia para "1", a velocidade tendesse para "o", corrigindo assim a inconsistência presente na formulação tradicional. Em termos algébricos, a "Lei de Bradwardine" exprime-se como:

$\mathrm{V}=\log (\mathrm{F} / \mathrm{R}) \quad \mathrm{ou} \quad \mathrm{V}=\log \mathrm{F}-\log \mathrm{R}$

Do ponto de vista da física intuitiva, o interesse destes primeiros esforços de algebrização do movimento é o de deslocarem o debate ente as teorias aristotélica e medieval para o terreno do conhecimento funcional (respeitante às relações 
contínuas entre diferentes variáveis), oferecendo uma outra forma de testar eventuais paralelos com a estrutura das representações espontâneas.

\section{O presente estudo}

A Teoria da Integração de Informação (TII) e da Medida Funcional (Anderson, 1981; Anderson, 1982) constitui um quadro natural para o estudo do conhecimento funcional. Tipicamente, uma tarefa de integração combina factorialmente diferentes variáveis independentes (no mínimo duas) e analisa o padrão de resultados decorrente da sua integração numa dimensão de resposta contínua (variável dependente), que pode ou não ser de natureza verbal. Três regras algébricas têm sido comummente encontradas em estudos com TII (aditiva, multiplicativa e de média), sugerindo a importância duma "álgebra cognitiva" para a estruturação funcional do conhecimento em diferentes domínios (Anderson, 1991). A operação de integração, realizada numa dimensão contínua (variando em grau ou quantidade), induz adicionalmente uma métrica sobre as variáveis a integrar (métrica subjectiva), que é parte integrante do modelo funcional. Numa tarefa de física ingénua organizada de acordo com metodologia da TII, a estrutura algébrica fornece assim um análogo da "lei física" e as "métricas subjectivas" um análogo das variáveis físicas intervenientes. Na medida em que os aspectos quantitativos são inseparáveis de uma base de conhecimento qualitativo, o estabelecimento de uma regra algébrica constitui também evidência favorável (ainda que não definitiva) à realidade psicológica das variáveis integradas através da regra.

O presente estudo propõe-se, através de uma tarefa de integração de informação na qual a "força motriz" e a "resistência do meio" são operacionalizadas e manipuladas como factores, verificar a existência de modelos de conhecimento funcional consistentes no domínio, e compará-los com as formulações algébricas peripatéticas e medievais.

\section{Método}

\section{Participantes}

33 Estudantes (30 F; 3 M) da Faculdade de Psicologia de Coimbra, com idades compreendidas entre os 18 e os 25 anos (Média: 20.12; DP: 4.86), participaram na experiência. Todos possuíam visão normal ou corrigida para o normal e desconheciam a natureza e propósito do estudo 


\section{Estímulos}

Os estímulos consistiram em representações esquemáticas, com vista de perfil, do lançamento de uma esfera no interior de um tubo horizontal contendo diferentes meios fluidos: ar, água, mel (ver Figura 1 A). A natureza do fluido em cada tubo era assinalada por uma cor característica (branco para o ar, azul para a água, castanho-mel para o mel). A esfera poderia ser colocada em movimento por um percutor de mola cuja tensão inicial se encontrava representada pelo grau de contracção de uma mola helicoidal, variado em quatro niveis (ver Figura 1 B). O tubo era intersectado por uma linha tracejada vertical numa de três localizações, correspondendo a outras tantas distâncias percorridas pela esfera (ver Figura 1 B).

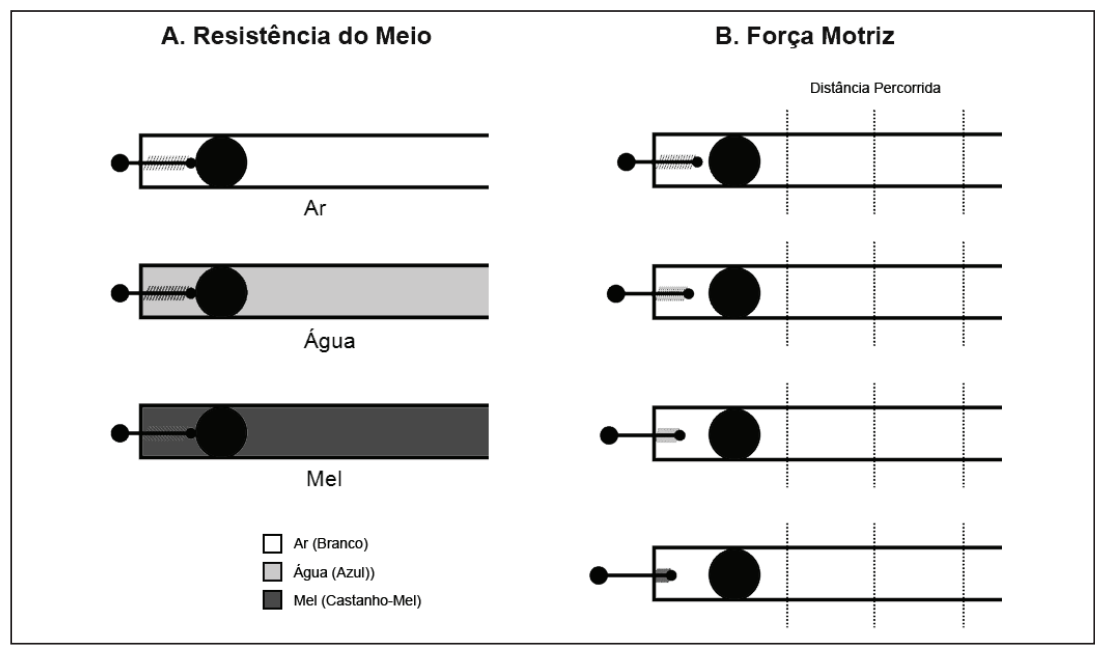

Figura 1. Representação esquemática dos estímulos: Painel A - Variação dos meios fluidos, em 3 níveis (a cada fluido foi feita corresponder um cor característica, identificada na legenda); Painel B - Variação da força aplicada pelo percutor de mola, em 4 niveis, e da distância percorrida pela esfera, em 3 níveis (linhas tracejadas)

\section{Desenho experimental e procedimento.}

A experiência obedeceu a um desenho factorial completo de medidas repetidas 4 (tensão do percutor/força motriz) ×3 (meio fluido/resistência) ×3 (distância percorrida).

Aos participantes era pedido que estimassem a velocidade da esfera no momento em que se atingisse a linha tracejada (assinalando uma distância percorrida) após ter sido lançada pelo percutor de mola. A resposta era expressa numa escala numérica de formato o-20, com as extremidades ancoradas nas expressões "esfera em repouso" e "máxima velocidade possibilitada pelo mecanismo". 
Os estímulos foram apresentados no centro de um monitor localizado a cerca de $60 \mathrm{~cm}$ dos participantes. Todos os aspectos da apresentação e aleatorização dos estímulos, bem como o registo das respostas, foram controlados com o programa SuperLab Pro 4.0.

\section{Resultados}

Análise gráfica. A Figura 2 apresenta o traçado factorial correspondente às relações entre força motriz (na abcissa) e resistência do meio (parâmetro da curva), com os resultados agregados através do factor distância percorrida (média das estimativas de velocidade na ordenada). O declive ascendente das curvas e o espaçamento vertical entre elas assinalam que ambos os factores têm efeitos, crescentes no primeiro caso, decrescentes (do meio menos denso ou resistente, o ar, para o meio mais denso ou resistente, o mel), no segundo. A divergência das curvas para a direita sugere a existência de uma interacção entre os dois factores e uma relação divisiva (ou, equivalentemente, multiplicativa: Fx1/R) entre ambos na determinação dos julgamentos de velocidade (cf. "teorema do leque linear" (linear fan) em Anderson, 1981; 1982; 1996). Este padrão factorial manteve-se inalterado nos gráficos traçados separadamente para cada um dos três níveis do factor distância percorrida (não apresentados), tratando-se assim de um resultado robusto

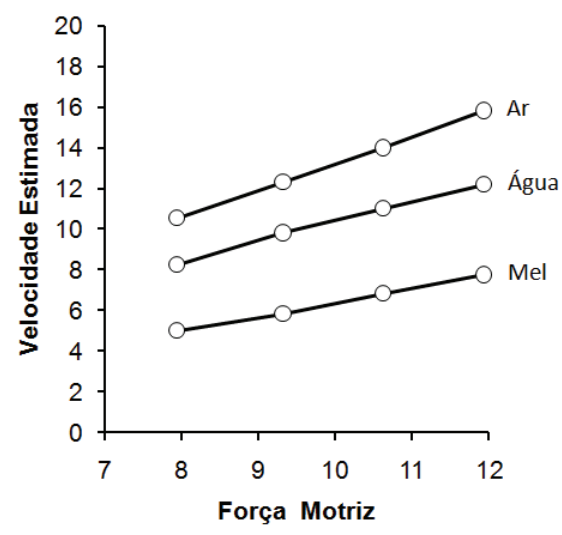

Figura 2. Gráfico factorial da força motriz (em abcissa) x resistência do meio (parâmetro das curvas). As estimativas de velocidade (na ordenada) foram agregadas através dos niveis do terceiro factor (distância percorrida)

Análise estatistica. As estimativas dos participantes foram submetidas a uma ANOVA de medidas repetidas 4 (força motriz) $\times 3$ (resistência do meio) $\times 3$ (dis- 
tância percorrida). Sempre que se verificou a violação da condição de esfericidade foi adoptada a correcção de Greenhouse-Geisser para os graus de liberdade. A análise estatística convergiu com as indicações resultantes da inspecção visual dos gráficos. Todos os factores apresentaram efeitos principais significativos: força motriz, $F(1.176,37.628)=46.173, p<.001$; resistência do meio, $F(1.436,45.954)$ $=54.51, p<.001$; distância percorrida, $F(1.079,34.53)=5.49, p=.023$. A interacção força motriz x resistência apresentou-se significativa, $F(3.519,112.62)=11.348, p<$ .001 , e localizada na componente linear-linear, $F(1,32)=25.453, p<.001$, o mesmo sucedendo com a interacção força motriz $x$ distância: $F(6,192)=2.667, p=.017$; contraste linear-linear: $F(1,32)=9.90, p=.004$.

Uma análise hierárquica de clusters (método: vizinho mais distante; medida: quadrado da distância euclidiana; estandardização: z, por caso) aplicada à mesma matriz de resultados revelou a presença de dois clusters fundamentais de participantes ( $C L$ 1: $n=21 ; C L$ 2: $n=9$ ) distinguindo-se quanto ao modo de funcionamento do factor distância percorrida. Enquanto no $\mathrm{CL} 1$ a distância apresenta um efeito principal significativo, $F(1.107,22.139)=12.469, p=.001$, e uma interacção significativa com a força motriz, $F(4.059,81.177)=3.829, p=.006$, no $C L 2$ não tem efeitos do ponto de vista estatístico $(F=1.217$, com um $p$ associado de .305 para o efeito principal; $F<1$ para a interacção). Quanto aos restantes factores, exibiram em ambos os clusters o mesmo padrão verificado na ANOVA principal: efeitos principais significativos (CL1: $p<.001 ; \mathrm{CL2:} p=.005)$ e um termo de interacção associado a uma componente linear-linear significativa $(\mathrm{CL} 1: F(1,20)=18.528, p$ $=.000 ; \mathrm{CL} 2: F(1,8)=7.062, p=.029)$.

Teste do modelo multiplicativo/divisivo. A assinatura estatística de uma relação multiplicativa/divisiva entre dois factores é a associação de uma componente bi-linear significativa (correspondendo ao perfil gráfico em leque linear) e de uma componente residual nula no termo da interacção (Anderson, 1982). A primeira destas condições foi documentada nas ANOVAs anteriores. A segunda condição foi testada com o módulo FM do pacote de programas CALSTAT (Weiss, 2006), revelando componentes residuais nulas $(F<1)$ para a interacção força $\times$ resistência, tanto na ANOVA geral como nas ANOVAs por cluster, e para a interacção força $x$ distância no CL1 (no CL2 esta interacção está ausente).

Tomando em conjunto as indicações fornecidas pela inspecção dos gráficos e pela análise estatística, os dados suportam consistentemente um modelo de integração divisivo entre a força motriz e a resistência do meio na determinação dos julgamentos de velocidade.

Velocidade $=($ Força motriz $) /($ Resistência do meio $)$ 
Este modelo é comum ao Cluster 1 e ao Cluster 2, no qual a variável distância percorrida não a presentou efeitos. No caso do Cluster 1 (maioritário), no entanto, o modelo completo deverá incluir a relação igualmente divisiva entre força motriz e distância percorrida, e ainda a relação aditiva, assinalada por uma ausência de interacção estatística e paralelismo no gráfico factorial, entre resistência do meio e distância percorrida, exprimindo-se algebricamente como:

Velocidade $=($ Força motriz) $/($ Resistência do meio+Distância percorrida $)$

\section{Discussão}

Os resultados encontrados estabelecem uma integração divisiva da "força motriz" e da "resistência do meio" (F/R) na determinação da velocidade do móvel, consistente com a tradução algébrica medieval da concepção aristotélica do movimento, mas não com a concepção do ímpeto de Philoponus, que advoga, diferentemente, uma integração subtractiva (F-R). Na medida em que a "rectificação" proposta por Bardwardine ao enunciado aristotélico $(\log (F / R))$ se pode exprimir como uma relação subtractiva entre os logaritmos de $F$ e de $R$ ( $\log F$ - log $R$ ), os resultados divergem igualmente desta revisão da concepção peripatética (avançada aliás não por razões de intuição, ou sequer empíricas, mas para garantir que $V=0$ quando $F=R$, diferentemente do que sucede na formulação divisiva: $V=1$ quando $F=R$ ).

No quadro dos paralelos históricos estabelecidos entre "física intuitiva" e as teorizações pré-newtonianas da mecânica, a física medieval do impetus, promovida por Jean Buridan (século XIV) e presente ainda nos primeiros escritos de Galileu, no início do século XVII (Kozhevnikov \& Hegarty, 2001), tem desempenhado um papel predominante, parecendo incorporar muitas das intuições reveladas pelos estudos de física ingénua (Clement, 1982; McCloskey, 1983a; 1983b). No entanto, a afinidade entre representações espontâneas da mecânica e o paradigma da física aristotélica tem sido igualmente defendida em estudos de física intuitiva (Champagne, Klopfer, L. \& Anderson, 1980; Watts \& Zylbersztajn, 1981). Porque ilustra uma afinidade da álgebra cognitiva com o quadro de pensamento aristotélico, o presente estudo poderia assim interpretar-se como um argumento em favor da analogia aristotélica contra a analogia do impetus.

Uma objecção a esta interpretação é a de que extrapola dos resultados obtidos (que estabelecem um modelo funcional particular, numa tarefa específica) para uma teoria espontânea do movimento susceptivel de entrar em ressonância com os corpora de princípios das físicas aristotélica ou do impetus. De um modo geral, dúvidas sobre a legitimidade de conferir à "física intuitiva" o estatuto de teoria (sistema geral e coerente de representações) têm sido expressas por vários auto- 
res. Por um lado, muitos dos erros documentados em estudos de física intuitiva não dispõem de um análogo histórico-epistemológico nos sistemas de crenças aristotélicos ou medievais (McDermott, 1984, Prescott \& Mitchelmore, 2005). Por outro, mesmo entre os proponentes da analogia aristotélica, a inconsistência das concepções ingénuas têm conduzido a descrevê-las como "pré-paradigmáticas" e localmente conciliáveis com soluções não aristotélicas (Champagne et al., 1980; Whitaker, 1983). Finalmente, a variabilidade das representações intuitivas em função de atributos específicos da tarefa constitui uma observação comum, apoiando mais a sua caracterização como "mini-teorias" (Millar \& Kragh, 1994), "fragmentos de conhecimento" (diSessa 1982) ou "modelos pastiche" (Collins and Gentner 1987) do que como um sistema de crenças articulado.

Esta última perspectiva é genericamente compatível com uma abordagem em termos de "modelos mentais" (Gentner \& Stevens, 1983). Em contraste com as teorias ingénuas ou populares (folk psychology), os modelos mentais foram propostos como "modelos de pequena escala" (Craik, 1947; Gentner, 2002) destinados a antecipar resultados e a suportar a acção em situações específicas. Um modelo mental integra elementos de conhecimento prévios (regra geral heterogéneos) de modo a conferir-lhes um papel funcional adaptado aos objectivos e situação presentes (Anderson \& Wilkening, 1991). Em virtude da sua natureza intrinsecamente adaptativa, não preexiste à situação e deve ser reconstruído na memória de trabalho a cada nova ocasião (Johnson-Laird, 1983). Um dos resultados fundamentais da investigação com modelos mentais é a possibilidade de dois ou mais modelos inconsistentes coexistirem num mesmo domínio (Gentner, 2002). Observações como as de Halloun e Hestenes (1985a; 1985b) de que os mesmos participantes, em diferentes situações, fazem uso de um misto de princípios aristotélicos, do impetus e newtonianos, disporiam aqui de um quadro de interpretação natural. Na mesma linha está a interpretação do impetus por diSessa (1988) como um construto associado a uma classe reduzida de problemas, e não como um princípio geral da física intuitiva.

O modelo de integração divisiva entre "força motriz" e "resistência do meio" pode entender-se, de modo similar, como um modelo mental da tarefa proposta, com a particularidade de se apresentar sob a forma de uma relação funcional (álgebra cognitiva). A circunstância de ilustrar uma integração regulada (e métrica) de todas as variáveis manipuladas (em particular no $\mathrm{CL}$ 1, maioritário) é contrária à tese de que a "física ingénua" reflecte basicamente limitações no processamento simultâneo de várias dimensões (Kaiser, Proffitt \& Anderson, 1985; Proffitt \& Gilden, 1989). Por outro lado, o perfil de diferenças individuais revelado pelos diferentes clusters de participantes é irredutivel tanto à matriz aristotélica como às diferentes variantes da teoria do impetus (cf. McCloskey, 1983). A ausência de efeitos da distância percorrida pelo móvel no CL2 é incompatível com a noção 
fundamental de dissipação do impetus, seja por acção espontânea, como em lbnSina (Avicena), seja por acção da resistência do ar ou da fricção, como em Buridan; é incompatível igualmente com o exercício da força resistiva atribuída ao meio na concepção aristotélica. Um funcionamento consistentemente subtractivo da "força motriz" (inassimilável também a qualquer das teorias consideradas) foi mesmo detectado num dos três participantes não incluídos nos Clusters 1 e 2 (o que, tratando-se de atrito devido a fluidos, poderia exprimir a noção fisicamente justificada de que a resistência oferecida por um fluido ao movimento cresce com a velocidade).

Globalmente, os resultados do presente estudo ilustram a utilidade da abordagem integrativa para o esclarecimento dos modelos cognitivos subjacentes à física intuitiva. Uma extensão natural desta abordagem consiste em utilizar, para além de julgamentos numéricos, outro tipo de respostas contínuas, directamente perceptivas (e.g., extrapolação de movimento: Ehrenstein, 1994) ou motoras (e.g., respostas de localização: cf. De Sá Teixeira \& Oliveira, 2008, 2010), no quadro duma mesma tarefa de integração. A comparação entre os padrões factoriais emergentes oferece uma via para a análise das dissociações e/ou comunalidades entre as representações de acção, perceptivas e cognitivas das relações físicas (Bertamini, Spooner, \& Hecht, 2004; Wilkening, 2002). Uma vantagem adicional das regras de integração algébrica, dado o papel primordial das relações funcionais na ciência moderna, é a possibilidade de aferir a correcção/incorrecção da física intuitiva por critérios não arbitrários (distintos, por exemplo, da exactidão quantitativa da resposta, ou do grau de sofisticação epistemológica que lhe é reconhecido, e centrados essencialmente na estrutura funcional do modelo cognitivo). Em conformidade, eventuais implicação pedagógicas da álgebra cognitiva devem procurar-se menos do lado dos contributos para a identificação e classificação epistemológica das "teorias alternativas" dos estudantes (Oliva, 2003) do que no quadro de um ensino da física centrado na aprendizagem regulada das formas de função (cf. Anderson, 1981, 298-299).

\section{Referências bibliográficas}

Anderson, N. H. \& Wilkening,, F. (1991). In N. H. Anderson (Ed.). Contributions to Information Integration Theory. Vol. III: Developmental (pp. 1-42). Hillsdale, N.J: Lawrence Erlbaum Associates.

Anderson, N. H. (1981). Foundations of Information Integration Theory. New York: Academic Press.

Anderson, N. H. (1982). Methods of Information Integration Theory. New York: Academic Press.

Anderson, N. H. (1991). Contributions to information integration theory (vols. 1, 2, 3). Hillsdale, NJ: Erlbaum. 
Bertamini, M., Spooner A., \& Hecht, H. (2004). The representation of naive knowledge about physics. In G. Malcolm (Ed.) Multidisciplinary Approaches to Visual Representations and Interpretations. Liverpool: Elsevier.

Caramazza, A., McCloskey, M., \& Green, B. (1981). Naive beliefs in "sophisticated" subjects: Misconceptions about trajectories of objects. Cognition, 9, 117-123.

Celeyrette, J. (2008). Bradwardine's rule: A mathematical law? In W. R. Laird \& S Roux (Eds). Mechanics and Natural Philosophy before the Scientific Revolution. Dordrecht: Springer.

Champagne, A. B., Klopfer, L. E. \& Anderson, J. H. (1980). Factors influencing the learning of classical Mechanics. American Journal of Physics, 48(12), 1074-1079.

Clagett, M. (1957). Science of Mechanics in the Middle Ages. Wisconsin: University of Wisconsin Press.

Clement, J. (1982). Students' preconceptions in introductory Mechanics. American Journal of Physics, 50(1), 66-71.

Collins, A, \& Gentner, D. (1987). How people construct mental models. In D. Holland and N. Quinn (Eds.). Cultural Models in Language and Thought (pp. 243-265). Cambridge, UK: Cambridge University Press.

De Sá Teixeira, N. A., de Oliveira, A. M., \& Amorim, M-A. (2010). Combined effects of mass and velocity on forward displacement and phenomenological ratings: A functional measurement approach to the momentum metaphor. Psicologica, 31, 659-676.

De Sá Teixeira, N. A., de Oliveira, A. M., \& Viegas, R. (2008). Functional approach to the integration of kinematic and dynamic variables in causal perception: Is there a link between phenomenology and behavioral responses? Japanese Psychological Research, 50, 232-241.

diSessa, A. (1988). Knowledge in pieces. In G. Forman and P. Pufall (Eds.). Constructivism in the Computer Age. (pp. 49-70) Hillsdale, NJ: Lawrence Erlbaum Associates.

diSessa, A. A. (1982). Unlearning Aristotelian physics: A study of knowledge-based learning. Cognitive Science, 6, 37-75.

Ehrenstein, W. H. Motion extrapolation and the Aubert-Fleischl phenomenon. Perception, 23 (suppl), 27 b.

Gentner, D. (2002). Mental models, Psychology of. In N. J. Smelser \& P. B. Bates (Eds.). International Encyclopedia of the Social and Behavioral Sciences (pp. 9683-9687). Amsterdam: Elsevier Science

Gentner, D., \& Stevens, A. L. (1983). Mental Models. Erlbaum: Hillsdale, NJ.

Halloun, I. A., \& Hestenes, D. (1985a). The initial knowledge state of college physics students. American Journal of Physics, 53, 1043-1055.

Halloun, I. A., \& Hestenes, D. (1985b). Commonsense concepts about motion. American Journal of Physics, 53, 1056-1065.

Jammer, M. (1957). Concepts of force. New York: Dover Publications

Jimoyiannis, A., \& Komis, V. (2001). Computer simulations in physics teaching and learning: A case study on students' understanding of trajectory motion. Computers and Education, 36, 183-204.

Kaiser, M. K., Proffitt, D. R., \& Anderson, K. (1985) Judgments of natural and anomalous trajectories in the presence and absence of motion, Journal of Experimental Psychology: Learning, Memory, and Cognition, 11, 795-803.

Kozhevnikov, M., \& Hegarty, M. (2001). Impetus beliefs as default heuristics: Dissociation between explicit and implicit knowledge about motion. Psychonomic Bulletin and Review, 8, 439-453. 
Laird, W. R., \& Roux, S. (2008). Mechanics and Natural Philosophy before the Scientific Revolution. Dordrecht: Springer.

McCloskey, M. (1983a). Intuitive Physics. Scientific American, 284, 114-122.

McCloskey, M. (1983b). Naive theories in motion. In D. Gentner \& A. Stevens (Eds.), Mental models (pp.299-324). Hillsdale, NJ: Lawrence Erlbaum.

McCloskey, M., \& Kohl, D. (1983). Naive physics: The curvilinear impetus principle and its role in interactions with moving objects. Journal of Experimental Psychology: Learning, Memory, \& Cognition, 9, 146-156.

McCloskey, M., Washburn, A., \& Felch, L. (1983). Intuitive physics: The straight-down belief and its origin. Journal of Experimental Psychology: Learning, Memory, and Cognition, 9, 636-649.

McDermott, L. C. (1984). Research on conceptual understanding in Mechanics. Physics Today, 37(7), 24-32.

McDermott, L. C. (1991). Millikan Lecture 1990: What we teach and what is learned -- Closing the gap, American Journal of Physics, 59, 301-315.

Millar, R., \& Kragh, W. (1994). Alternative frameworks or context-specific reasoning? Children's ideas about the motion of projectiles. School Science Review, 75(272), 27-34.

Oliva, J.M. (2003), The structural coherence of students' conceptions in mechanics and conceptual change, International Journal of Science Education 25: 539-561.

Prescott, A. E. \& Mitchelmore, M. (2005). Student misconceptions about projectile motion. In P. Clarkson, A. Downton D. Grpnn, M. Horne, A. McDonough, R. Pierce, and A. Roche (Eds.) Building Connections: research, theory and practice (pp. 633-640). Sydney: MERGA.

Proffitt, D. R. (1999). Naive physics. In R. Wilson \& F. Keil (Eds.). The MIT encyclopedia of the cognitive sciences. Cambridge, MA: MIT Press.

Proffitt, D. R., \& Gilden, D. L. (1989). Understanding natural dynamics, Journal ofExperimental Psychology: Human Perception and Performance. 15, 384-393.

Rezende, F. \& Barros, S. S. (2001). Teoria Aristotélica, Teoria do Impetus ou Teoria Nenhuma: um Panorama das Dificuldades Conceituais de Estudantes de Física em Mecânica Básica. Revista Brasileira de Pesquisa Em Educação em Ciências, 1( 1), 43-56.

Thakkar, M. (2007). The oxford calculators. Oxford Today, 19(3), 24-26.

Trowbridge, D. \& McDermott, L. (1980). Investigation of student understanding of the concept of velocity in one dimension. American Journal of Physics, 48, 1020-1028.

Viennot, L. (1979). Spontaneous reasoning in elementary dynamics. European Journal of Science Education, 1, 205-221.

Watts, M. \& Zylbersztajn, A. (1981). A survey of some childrens' ideas about force. Physics Education, 16(6), 360-365.

Whitaker, R. (1983). Aristotle is not dead: Student understanding of trajectory motion. American Journal of Physics, 51(4), 352-357.

Hecht, H., \& Proffitt, D. R. (1995). The price of expertise: Effects of experience on the waterlevel task. Psycological Science, 6(2), 90-95.

Proffitt, D. R., \& Gilden, D. L. (1989). Understanding natural dynamics. Journal of Experimental Psychology: Human Performance and Perception, 15, 384-393. 


\section{Aristóteles versus Philoponus: a functional approach to the intuitive physics of projectiles}

Intuitive physics is a popular field of research within cognitive psychology which addresses the common-sense beliefs about the physical world, particularly about classical mechanics. An influential stream of research argues for the striking resemblance between common-sense beliefs and those embodied in pre-Newtonian theories, such as the medieval physics of impetus. In this study, two early algebraic proposals for the relation between the force applied to an object, the resistance to its motion and its resulting velocity are compared, the first one derived from Aristotle's Physics, the other set forth by Philoponus, in the $\mathrm{V}^{\mathrm{lth}}$ century. To this end, the methodology of Information Integration Theory and Functional Measurement was used, revealing a dividing cognitive integration rule in agreement with Aristotle's proposal. The significance of this finding is discussed through a suggested link with the mental models framework. The potential contribution of cognitive algebra to the field of intuitive physics is also briefly pinpointed.

KEY-WORDS: Intuitive Physics, Naive Physics, Impetus, Mental Models, Information Integration Theory, Cognitive Algebra.

\section{Aristote versus Philoponus: une approche fonctionnelle de la physique intuitive des projectiles}

En tant que domaine de recherche, la physique intuitive s'occupe des croyances spontanées au sujet de la structure et du fonctionnement du monde physique, et en particulier des événements mécaniques. Des parallèles étroits ont été suggérés entre ces croyances et les principes soutenus dans le cadre des théories physiques précédant celle de Newton, telles que la physique aristotélique ou la physique médiévale de l'impetus. On compare dans cette étude deux essais historiques de mathématisation des relations entre la vélocité d'un objet, la force avec laquelle il est lancé, et la résistance du milieu à son mouvement, le premier desquels dérivé des écrits d'Aristote (siècle IV a. C.) et le deuxième du à Philoponus (VI ème siècle). La méthodologie de la Théorie d'Intégration d'Information et de la Mesure Fonctionnelle à été employée à cet effet. Une règle cognitive de division a pu être établie, en accord avec la proposition aristotélique. On discute la portée de ce résultat, moyennant un rapport suggéré avec le cadre des modèles mentales. L'apport potentiel de l'algèbre cognitive dans le domaine de la physique intuitive est aussi brièvement discuté.

MOTS-CLÉS: Physique Intuitive, Physique Naïve, Impetus, Théorie de l'Intégration de l'Information, Algèbre Cognitive. 